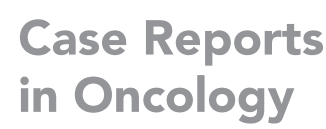

Case Reports

in Oncology

This article is licensed under the Creative Commons Attribution-NonCommercial 4.0 International License

\title{
Male Breast Carcinoma after Irradiation and Long-Term Phenothiazine Exposure: A Case Report
}

\author{
Ragnar Hultborn ${ }^{a} \quad$ Toshima Z. Parris $^{b} \quad$ Khalil Helou $^{\mathrm{b}} \quad$ Åke Borg ${ }^{\mathrm{c}}$ \\ Shahin De Lara ${ }^{d}$ Anikó Kovács ${ }^{d}$

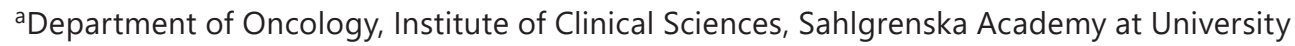 \\ of Gothenburg, Gothenburg, Sweden; bepartment of Oncology, Institute of Clinical \\ Sciences, Sahlgrenska Cancer Center, Sahlgrenska Academy at University of Gothenburg, \\ Gothenburg, Sweden; 'Division of Oncology, Department of Clinical Sciences, Lund \\ University, Lund, Sweden; dDepartment of Clinical Pathology, Sahlgrenska University \\ Hospital, Gothenburg, Sweden
}

\section{Keywords}

Male breast cancer - BRCA1 - Schizophrenia - Irradiation · Phenothiazine ·

Hyperprolactinemia

\section{Abstract}

We present a young male patient with breast cancer having several risk factors likely acting in consort: irradiation of the breast for gynecomastia in adolescence and a life-long administration of phenothiazine for schizophrenia from the age of 16 years, with elevated serum prolactin level resulting in breast cancer development 24 years after irradiation.

\section{Introduction}

Male breast cancer is a rare disease primarily found in elderly men that accounts for approximately $0.5-1 \%$ of breast carcinomas diagnosed in female counterparts in Europe and the US [1]. The pathogenesis of male breast cancer has been ascribed to intrinsic genetic factors (e.g., XXY Klinefelter syndrome and BRCA2 mutations) and various extrinsic factors, such as irradiation, hormonal perturbations from phenothiazine, estrogen treatment or liver dysfunction [1].

We describe an unusual case, breast cancer in a young male, with several possibly interacting predisposing factors which will be discussed. 


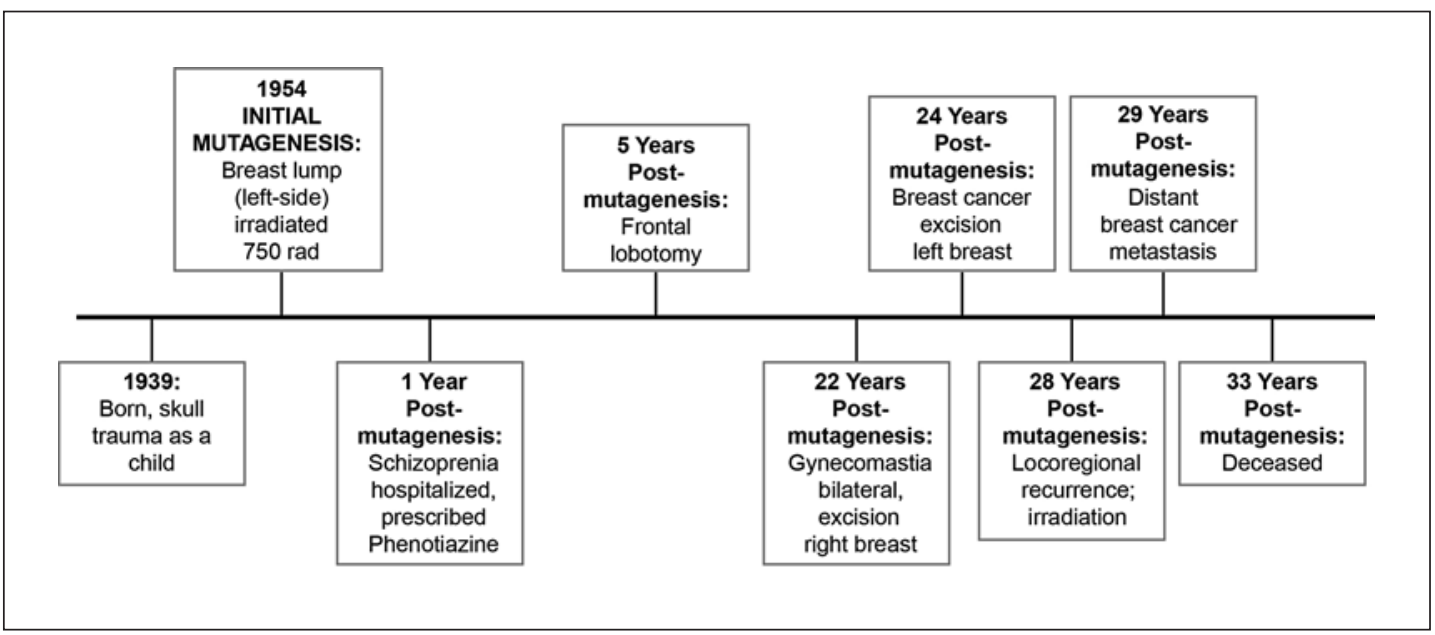

Fig. 1. Timeline showing the consecutive events during the patient's life.

\section{Case Presentation}

The male patient was born in 1939, and the events over time are shown in Figure 1. He had skull traumas twice as a child. Both parents and siblings had been treated for psychiatric diseases. No information on breast cancer heredity was available. At the age of 14 , a left-sided tender breast swelling was treated by X-irradiation, not practiced today $(165 \mathrm{kV}, 4 \mathrm{~mm}$ aluminum) at a total dose of 750 rad over 10 days with remission of the swelling. A year later, at the age of 16, he was hospitalized under the diagnosis of fulminant schizophrenia. He received heavy phenothiazine (chlorpromazine) treatment, insulin coma as well as electroconvulsive treatment with insufficient outcome. He underwent a frontal lobotomy 5 years later, but he had to continue life-long with heavy phenothiazines and was hospitalized.

At the age of 37, bilateral gynecomastia developed, likely from the phenothiazine medication. The dominant lump in the right breast was surgically removed and it was histopathologically diagnosed as gynecomastia without atypia. Two years later, at the age of 39 , the lump in the previously irradiated left breast started to grow. A simple mastectomy, without axillary lymph node dissection was performed. Microscopic examination revealed an invasive ductal breast carcinoma, radically excised without any postoperative treatment. Four years later, an extensive locoregional recurrence was diagnosed, but no distant metastases. An axillary lymph node was removed for verification of the breast cancer metastasis with steroid receptor analysis and ploidy determination.

The patient had locoregional radiotherapy, $8 \mathrm{MeV}$ electrons to the internal mammary lymph nodes, as well as to the previously partly irradiated thoracic area and with photons to the axillary and supraclavicular nodes. It was not possible to avoid phenothiazines during radiotherapy due to an aggressive confusion. The patient developed an unusually intense dark brown skin reaction. Tamoxifen, $20 \mathrm{mg}$ daily was prescribed but was stopped after 18 months due to thrombocytopenia, which normalized after treatment cessation. A year later, the patient developed widespread distant metastases. Chemotherapy and further hormonal treatments were considered contraindicated and he died without antitumoral treatment 4 years later at the age of 48 .

\section{Laboratory Findings}

In 1976, histopathology after surgery of the right breast lump disclosed a gynecomastia of the fibrous type with no signs of atypia or cancer. In 1978, histopathology after simple 


\section{Case Reports in Oncology}

\begin{tabular}{l|l}
\hline Case Rep Oncol 2020;13:956-961 \\
\hline DOI: 10.1159/000509074 & $\begin{array}{l}\text { @ 2020 The Author(s). Published by S. Karger AG, Basel } \\
\text { www.karger.com/cro }\end{array}$ \\
\hline
\end{tabular}
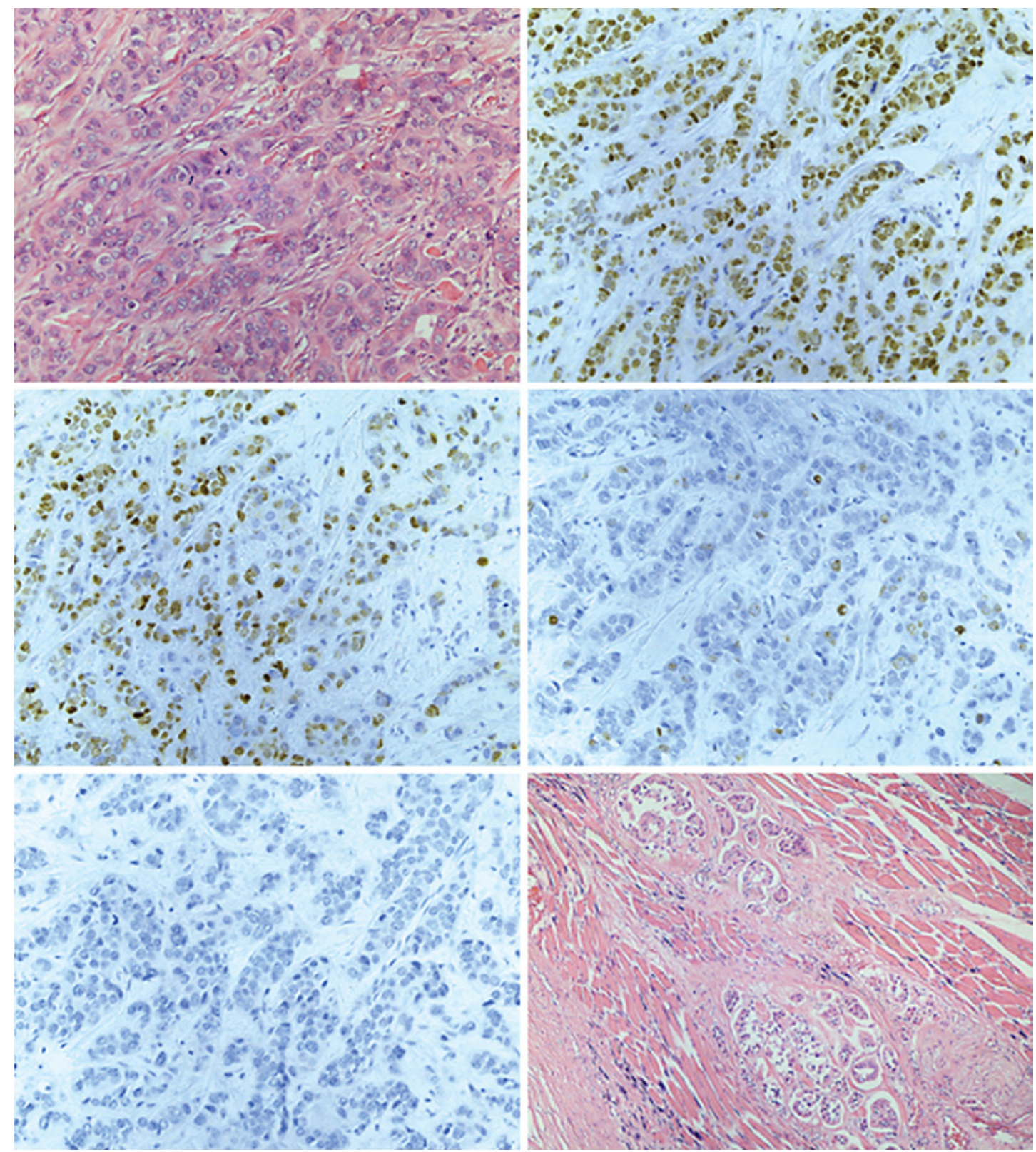

Fig. 2. Invasive ductal carcinoma. HE. $\times 200$ (top left). Androgen receptor immunostaining, $\times 200$ (top right). Progesterone receptor immunostaining, $\times 200$ (middle left). Ki-67 proliferation marker immunostaining, $\times 200$ (middle right). HercepTest for HER2 immunostaining (score 0), $\times 200$ (bottom left). Local recurrence of invasive ductal carcinoma in musculus pectoralis major, $\times 40$ (autopsy material; bottom right).

mastectomy of the left breast revealed two clearly separated foci of a poorly differentiated invasive ductal carcinoma. In 1982, cytology verified a metastatic growth of invasive ductal carcinoma in an axillary lymph node followed by subsequent removal.

Steroid hormone receptors were quantified by the dextran-coated charcoal technique, estrogen receptor (ER) was highly positive ( $3.22 \mathrm{fmol} / \mu \mathrm{g}$ DNA or $93 \mathrm{fmol} / \mathrm{mg}$ protein), while no progesterone receptors (PR) were detected. 
Flow cytometry of the lymph node metastasis disclosed polyploidy (DNA index 2.0, 3.8 and 5.6) with an S-phase fraction of 7-9\%. Peripheral blood karyotyping disclosed a normal XY male karyotype.

Serum prolactin was measured three times in 1982 during ongoing phenothiazine treatment but prior to tamoxifen, showing high values $(1,007,1,580$ and 1,058 $\mathrm{mU} / \mathrm{L})$ compared to normal values at the laboratory (range 35-350 mU/L).

Histopathological re-evaluation of the archival formalin-fixed paraffin-embedded primary tumor material was performed in 2019 verifying an invasive ductal carcinoma with a BRE score (Bloom-Richardson-Elston) of 8 (tubulus formation 3, nuclear pleomorphism 3, mitotic activity 2). Immunohistochemical examination of the invasive breast carcinoma showed $100 \%$ positivity in ER staining, 70\% positivity in PR staining and 95\% positivity in androgen receptor staining. HER2 status was scored as negative (score 0) in the HercepTest and prolactin receptor was negative (Fig. 2). Autopsy material disclosed the same histology, but immunohistochemistry was totally negative for all biomarkers, likely due to autolysis.

BRCA1 and BRCA2 screening, using single-molecule molecular inversion probes and Illumina sequencing of tumor DNA, revealed a novel pathogenic BRCA1 sequence variant (c.1020dupA, p.(Asp341Argfs*5)).

This variant was confirmed by Sanger sequencing but was not present in the DNA from the gynecomastia specimen, thus representing a somatic mutational event possibly caused by previous radiotherapy.

The tumor was steroid hormone receptor positive, an unexpected finding since most tumors with BRCA1 mutation are triple negative. Further, most ER- and PR-positive tumors are prolactin receptor positive [2]. This might represent an unusual situation or a falsenegative result from the age of the tumor material.

\section{Discussion}

The case presented includes a number of interacting potential intrinsic and extrinsic factors influencing the evolution of the rare cancer he developed.

Intrinsic factors that prevail in this case is schizophrenia itself. Several but conflicting reports indicate that the incidence of most malignancies except breast cancer is lower among those with schizophrenia than in the general population [3-6], but a recent meta-analysis does not support this view [7]. Those claiming that genetic factors are associated with a lower cancer incidence refer to the findings that siblings and parents also show a lower cancer incidence than the population in general $[3,8,9]$. Zhuo et al. [10] review various molecular mechanisms accounting for a suppressed cancer development. However, confounding factors among these patients obscure the conclusions. The discrepancy between a lower or higher risk of cancer in this special patient group can be due to differences in cancer types, antipsychotic medication, lifestyles (often a sedentary lifestyle, neglecting a balanced diet, abusing alcohol, tobacco smoking), ethnicity and gender $[4,5]$, as well as due to different sampling techniques of the populations studied [11].

Other intrinsic risk factors such as an XXY karyotype or germline BRCA1/2 mutations were not present in this case.

Among the exogenous factors, the most important one is the exposure to ionizing radiation $[12,13]$. No data on the consequences of radiotherapy for adolescent gynecomastia have been published to the best of our knowledge, but it is reasonable to assume an increased risk for cancer initiation in the proliferative tissue.

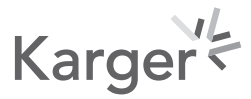


Among schizophrenic patients, the antipsychotic treatments, in our case heavy and lifelong phenothiazines (chlorpromazine), result in profound hyperprolactinemia [14, 15]. Possibly the early head traumas added, tentatively also to the adolescent gynecomastia [16]. Antipsychotics may themselves influence the risk of malignancy, but due to the influence on prolactin secretion, the results are difficult to interpret since prolactin is considered a promotor for breast cancer progression [17-19].

The complex interactions, positive and negative, of schizophrenia itself, antipsychotics themselves and the tumor-specific effects from the induction of hyperprolactinemia explain the divergent results concerning cancer incidence among schizophrenics.

Further, the lifestyles among schizophrenics are different from the general population, for example, smoking and obesity as in our case, favoring cancer development. Diagnostic denial might underestimate true incidence.

\section{Conclusion}

This case illustrates a schizophrenic patient with irradiation initiation, acting together with promotors, that is, hyperprolactinemia was induced possibly by the early head trauma and certainly by phenothiazine long-term medication, resulting in male breast cancer with a latency of 24 years after induction in a young non-BRCA1/2-mutated XY male.

\section{Acknowledgement}

The study was supported by a grant from the Gustaf V's Jubilee Foundation. We would like thank Mr. Abbas Rezazadeh and Mr. Bora Todorovic for their invaluable help with retrieving the formalin-fixed paraffin embedded blocks containing the patient's biopsy and autopsy samples.

\section{Statements of Ethics}

The study was approved by the Local Scientific Committee in Gothenburg (D-No.: 768-14). The requirement for informed consent was waived by the ethics committee since patient materials were stripped from direct subject identifiers.

\section{Conflict of Interest Statement}

No conflicts of interest from any of the authors.

\section{Author Contributions}

A.K., R.H.: concepts; A.B., K.H., T.Z.P.: genetics; S.D.L.: immunohistochemistry. 


\section{References}

1 Fentiman I. Male Breast Cancer. Springer International Publishing AG; 2017.

2 Ormandy CJ, Hall RE, Manning DL, Robertson JF, Blamey RW, Kelly PA, et al. Coexpression and cross-regulation of the prolactin receptor and sex steroid hormone receptors in breast cancer. J Clin Endocrinol Metab. 1997; 82(11):3692-9.

3 Ji J, Sundquist K, Ning Y, Kendler KS, Sundquist J, Chen X. Incidence of cancer in patients with schizophrenia and their first-degree relatives: a population-based study in Sweden. Schizophr Bull. 2013;39(3):527-36.

4 Chou FH, Tsai KY, Su CY, Lee CC. The incidence and relative risk factors for developing cancer among patients with schizophrenia: a nine-year follow-up study. Schizophr Res. 2011;129(2-3):97-103.

5 Grinshpoon A, Barchana M, Ponizovsky A, Lipshitz I, Nahon D, Tal O, et al. Cancer in schizophrenia: is the risk higher or lower? Schizophr Res. 2005;73(2-3):333-41.

6 Mortensen PB. Neuroleptic treatment and other factors modifying cancer risk in schizophrenic patients. Acta Psychiatr Scand. 1987;75(6):585-90.

7 Zhuo C, Triplett PT. Association of Schizophrenia with the Risk of Breast Cancer Incidence: A Meta-analysis. JAMA Psychiatry. 2018;75(4):363-9.

8 Lichtermann D, Ekelund J, Pukkala E, Tanskanen A, Lonnqvist J. Incidence of cancer among persons with schizophrenia and their relatives. Arch Gen Psychiatry. 2001;58(6):573-8.

9 Catts VS, Catts SV, O'Toole BI, Frost AD. Cancer incidence in patients with schizophrenia and their first-degree relatives - a meta-analysis. Acta Psychiatr Scand. 2008;117(5):323-36.

10 Zhuo C, Wang D, Zhou C, Chen C, Li J, Tian H, et al. Double-Edged Sword of Tumour Suppressor Genes in Schizophrenia. Front Mol Neurosci. 2019;12:1.

11 Whitley E, Batty GD, Mulheran PA, Gale CR, Osborn DP, Tynelius P, et al. Psychiatric disorder as a risk factor for cancer: different analytic strategies produce different findings. Epidemiology. 2012;23(4):543-50.

12 Kamran SC, Berrington de Gonzalez A, Ng A, Haas-Kogan D, Viswanathan AN. Therapeutic radiation and the potential risk of second malignancies. Cancer. 2016;122(12):1809-21.

13 Eldar S, Nash E, Abrahamson J. Radiation carcinogenesis in the male breast. Eur J Surg Oncol. 1989;15(3): 274-8.

14 Goodnick PJ, Rodriguez L, Santana O. Antipsychotics: impact on prolactin levels. Expert Opin Pharmacother. 2002;3(10):1381-91.

15 Haddad PM, Wieck A. Antipsychotic-induced hyperprolactinaemia: mechanisms, clinical features and management. Drugs. 2004;64(20):2291-314.

16 Olsson H, Ranstam J. Head trauma and exposure to prolactin-elevating drugs as risk factors for male breast cancer. Journal Natl Cancer Inst. 1988;80(9):679-83.

17 Hendouei N, Saghafi F, Shadfar F, Hosseinimehr SJ. Molecular mechanisms of anti-psychotic drugs for improvement of cancer treatment. Eur J Pharmacol. 2019;856:172402.

18 Clevenger CV, Furth PA, Hankinson SE, Schuler LA. The role of prolactin in mammary carcinoma. Endocr Rev. 2003;24(1):1-27.

19 Johnston AN, Bu W, Hein S, Garcia S, Camacho L, Xue L, et al. Hyperprolactinemia-inducing antipsychotics increase breast cancer risk by activating JAK-STAT5 in precancerous lesions. Breast Cancer Res. 2018;20(1): 42. 